\title{
The pathogenesis and prevention of corneal graft melting after keratoplasty.
}

\author{
Binbin Jin, Xiuming Zhu* \\ Eye Center, School of Medicine, Affiliated Second Hospital, Zhejiang University, 88 Jiefang Road, Hangzhou \\ 310009, China
}

\begin{abstract}
Keratoplasty is one of the most important treatments of corneal disease. Corneal graft melting is a severe complication that may occur in various types of keratoplasy. With various biomaterials and synthetic materials as substitutes for alternative corneal allograft donors being used in the clinic, there is a rising trend towards corneal graft melting after keratoplasy with these new materials. Corneal graft melting can be caused by many reasons. The review summarizes literature on the incidence, pathogenesis and prognosis of corneal graft melting after keratoplasty. Our results may help to develop suitable management to prevent corneal graft melting, and improve the outcomes of keratoplasty.
\end{abstract}

Keywords: Corneal graft melting, Keratoplasty, Incidence.

Accepted on October 24, 2017

\section{Introduction}

It is generally known that common complications of keratoplasty include immune rejection, corneal graft melting, secondary glaucoma, recurrence of primary disease, etc [1]. Among them, corneal graft melting is a severe complication that can occur in various types of keratoplasy. Corneal graft melting may lead to scarring, irregular astigmatism, photophobia, decreased vision, and even endophthalmitis [2]. Corneal graft melting is related to many factors such as graft materials, the operative type, and postsurgical nursing methods. Among those factors, the clinical application of alternative materials for corneal allograft donor deserves enough attention.

The review summarizes various types of literature on incidence, pathogenesis, prognosis and treatment of corneal graft melting after keratoplasty.

\section{Corneal Graft Melting in Various Types of Keratoplasy}

Many Studies showed that corneal graft melting is one of very serious complications in keratoplasty. Moreover, it has been reported that all types of keratoplasty may happen infectious and sterile corneal graft melting with different incidence.

\section{Infectious corneal graft melting}

Infection-related corneal graft melting is thought to have two forms of development: (1) Infection directly results in corneal graft melting. (2) Corneal graft melting is an aseptic process subsequent controlled infection.

A study reported that $7.84 \%$ (4 eyes) patients with high-risk fungal keratitis underwent penetrating keratoplasty happened corneal graft melting. $5.88 \%$ patients were controlled by treatments, and one suffered severe endothelial cell loss leading to replace corneal graft. Recurrence of primary disease occurred in 6 cases $(11.76 \%)$, with cornea ulcer. $7.84 \%$ patients were controlled, and 3.92\% patients developed endophthalmitis and underwent enucleation [2].

In $18.2 \%$ patients, corneal graft melting occurred after surgical treatment, which is common in penetrating keratoplasty and ulcer perforation. Some cases that are caused by local bacterial or fungal infections occur in the peripheral of implants [3]. Fungal infection has been proved that it commonly occurs in LKP and PKP. Candida albicans infection is in the majority [4]. Jastaneiah et al. reported that fungal infection occurred in 124 patients after ophthalmic surgery between 1984 and 2004 [5]. In another case corneal graft melting occurred because of the fungal infection, and was performed repeat keratoplasy, since the local antifungal drugs are not effective in treatment previous studies found that there are serious complications in keratoprosthesis. Most KPro complications are associated with ocular surface inflammation and ocular surface dryness and it is estimated that the incidence of infection-related corneal graft melting is approximately $51 \%[6,7]$.

Corneal graft melting ranges from corneal planting bed to corneal graft including device. It commonly happens in the area of the infection-induced corneal epithelial defect or corneal infiltrate Corneal graft melting after allogeneic keratoplasty has not been reported in detail [8-11].

\section{Sterile corneal graft melting}

Li et al. [12] analyzed postoperative complications in 274 patients with deep lamellar keratoplasty. Among them, 62 patients had various complications, and the incidence is $22.6 \%$. Bad corneal epithelial healing and sterile corneal stromatolysis occurred in 5 eyes $(1.82 \%)$. The results showed that the incidence of corneal graft melting in DALK is much less than in PKP.

Green [13] had found two cases of sterile corneal graft melting after undergoing descemet stripping endothelial keratoplasty (DSEK) with Sjogen Syndrome. The study reported persistent 
corneal epithelial defect. Finally, the visual acuity of the patients was poor, but the ulceration was resolved by using immunodepressants, glucocorticoid and so on [13]. Studies reported that the main reason of corneal graft failures with rheumatoid arthritis was melting after PKP, which accounted for $80 \%$.

Tissue-engineered corneal equivalents (TECs) have been a potential material substitute for corneal xenotransplantation $[12,13]$. Acellular porcine cornea is commonly used scaffolds for tissue-engineered cornea. Zhang et al. [14] found that 4 cases had corneal graft melting among 47 patients who implanted acellular porcine corneal stroma (APCSs). But those corneal grafts turned to corneal epithelialization. A Study of rabbit model with Acellular Porcine Corneal Stroma (APCS) and keratocytes showed that corneas in APCS group were thinner than normal cornea, which indicating that most of the scaffold finally would be degraded leading to sterile melting [15]. Liu et al. [16] used cellular ostrich corneal stroma as scaffold of tissue-engineered cornea comparing with porcine cornea, and the results showed the better property of ostrich corneal stroma. But part of the scaffold still had dissolved.

Patients are mostly complicated corneal blindness prior to KPro implantation, especially corneal chemical injury with poor ocular surface condition [17]. Corneal graft melting is the most common complication of KPro implantation, with the incidence of up $6 \%$ to $25 \%[18,19]$. However, recent reports stated that the rate of corneal graft melting is about $1.4 \%$ and $17 \%$ after keratoplasty, and usually occurs within two months, which incidence was much lower than the previous data. The survival rate of the high-risk keratoplasty is less than $35 \%$ [20-23].

In a study, Boston type 1 keratoprosthesis was performed in $16.4 \%$ cases and implanted suffered postoperative device melting, while Robert reported 3\% patients underwent KProrelated corneal graft melting within first 3 years. With a follow-up period ranging from 21 to 101 months, 63 patients implanted Dohlman-Doane keratoprosthesis, 29\% of who underwent device melting [24]. It was consistent with the results of Chan findings, who found that 18 patients (20 eyes) developed corneal graft melting among 110 patients (128 eyes) with Boston type 1 keratoprosthesis. The onset is earlier in the patients with conjunctival defect and infectious keratitis, and the melting rate of the former is $33 \%$ [8].

Although the incidence of corneal graft melting is lower than that of other postoperative complications, its consequences are more severe. Therefore, it is necessary to learn more about the factors in order to find better prevent and cure the corneal graft melting.

\section{Pathogenesis}

Many factors that cause corneal graft melting after keratoplasy and most of them have a common mechanism, own unique pathway, which eventually lead to the occurrence and development of corneal graft melting.

\section{Abnormal corneal state}

Potential immune crisis of cornea: Keratitis, corneal burn, surgery and other factors can elicit immune response. Immune system plays a very important role in postoperative corneal graft melting. The immune inflammatory cells (including polymorphonuclear neutrophils, lymphocytes, Langerhans cells etc.) and immune components (such as cytokines, adhesion molecules, chemokines) interact and form a complex immune inflammatory network. Although inflammatory cells clear and inactivate toxic substances, which is important for the body self-protection and beneficial to corneal wound healing. However, inflammatory cell products often specifically damage tissue that mediates the abnormal pathological changes of corneal diseases. Damaged epithelium will release proinflammatory cytokine to increase polymorphonuclear neutrophils, which results in the corneal graft melting. Many experiments showed polymorphonuclear neutrophils can release a lot of collagenase to dissolve collagen fiber, and then develop to ulceration and further deterioration. On the other hand, epithelial cells can regulate the secretion of collagenase, and active latent collagenase released by keratocyte and polymorphonuclear neutrophils [25]. It suggests that infiltration of a large number of leukocytes is one of main reasons of aseptic corneal graft melting and perforation $[25,26]$. With vascularization of corneal planting bed, the corneal interacts more with the immune cells and immune components.

\section{Corneal edema}

Researchers found that corneal edema may also cause corneal graft melting. There are several evidences explained that corneal edema eventually leads to corneal graft melting.

Firstly, corneal edema makes corneal tissue loose. Blood vessels in the corneal limbus invade into swelling and loose stroma that may cause malnutrition of the graft leading to melting. Furthermore, when the postoperative inflammation decreased and the corneal edema subsides, it also leads to suture loosening. It is easy for secretions to adhere loosening suture resulting in secondary infection and immune rejection. Both factors can cause corneal graft melting through immune reaction [27].

Jing et al. [28] analyzed 42 patients (47 eyes) for complications of Descemet's stripping automated endothelium keratoplasty (DSAEK). Graft shrinkage results in the edge tilt of the graft, and the planting bed around the graft is renewed. The nerve and keratocytes are difficult to grow into the graft, and eventually the graft spontaneously melts due to lack of nutrition and innervation.

Corneal stromal edema causes epithelial cells to lose good growth microenvironment, which affects the growth of corneal epithelial cells leading to conjunctivalization and corneal scarring. Because of long-term corneal edema and degeneration, coupled with inflammation, the corneal graft eventually develops melting and perforation [29]. 


\section{Clinic Pathology}

\section{Ocular diseases}

Dry eye: The characteristics of patients with dry eye are low tear secretion, rapid tear evaporation and poor tear film stability. Patients with traumatic or congenital eyelid defect, hyperthyroidism, trigeminal nerve paralysis and other reasons for lagophthalmos often have corneal dryness, corneal epithelial defect and even corneal ulcer, which may affect the survival of corneal graft after surgery [14]. It has found that tear break-up time (BUT) shortened and tear film was unstable after transplantation. The mechanisms are as follows: (1) the corneal nerve is mechanically damaged by the cornea ring drill. A study on the follow-up of 71 patients with PKP, through the observation of nerve regeneration, found that nerve was not observed in the corneal graft of all patients within 1 month after the operation. The nerve grew into the implant by 3 to 6 months after the operation, and the nerve growth in twelve months was the same as that in six months after operation. In addition, Niederer et al. [30] revealed that the nerve fiber density was not restored to normal levels after 40 years in patients with PKP. The morphology of regenerated nerve fibers is distorted, and the nerve thickness is different from the normal nerve. Because of the slow recovery of nerve, or trigeminal nerve paralysis, congenital corneal sensation, it easily leads to lack of nerve nutrition in the cornea, impaired corneal sensation and decreased corneal sensitivity. Then normal blinking reflex reduced, tear distribution is uneven, and leads to the corneal epithelial defect and bad healing. The epitheliums of the graft are shed, and the collagenase will dissolve the cornea. (2) Surgery will affect the regularity of the cornea. Recovering the smooth ocular surface takes time and surgery affects the mucins secretion of the conjunctival goblet cells, and tears stay unsteadily on the corneal surface. (3) Lack of corneal epithelial protection makes it easily to change corneal collagen caused by the use of hormones and immunosuppressants after surgery, which aggravates corneal graft melting [31,32]. Some researchers proved that the epithelial healing time of patients with Shirmmer test $<5 \mathrm{~mm}$ was longer, resulting in a high risk of necrosis and melting of the graft $[33,34]$.

Recurrence of primary disease: Unskilled surgical techniques are, and tiny lesions may lead to incomplete removal of the lesion, resulting in the recurrence of the primary disease. A recurrence of herpes simplex keratitis and fungal keratitis after corneal transplantation are also reported $[35,36]$.

Once recurrence of primary disease and other postoperative infection occurs, a large number of inflammatory cells gather in the cornea. For example, fungal infiltrate and propagate in the corneal stroma, release valinomycin and proteolytic enzymes leading to tissue necrosis, and develop towards corneal posterior lamellar into infectious corneal graft melting, corneal perforation, hypopyon and enucleation [37]. At the same time, expression of MMPs is increased that can degrade corneal stroma, and then lead to the corneal graft melting. In addition, MMPs promote angiogenesis and its mechanism is that elevated MMPs damage the cornel epithelial cells. It develops to corneal ulcer when corneal epithelial defect persists for a long period. Eventually the blood vessels entre the cornea, and then release various inflammatory factors [38].

Mooren's ulcer (MU) is a presumed autoimmune disease of unknown etiology. Most of these patients have severe corneal injury, accompanying with abundant new blood vessels in ocular surface [39]. In MU patients who had lamellar keratoplasy, the level of soluble protein in the corneal stroma and autoantibody soluble antigen in the patients' sera abnormally increased [40]. Studies have put forward that denatured soluble protein diffused to the limbus via edematous and loose corneal stroma, and stimulates the proliferation of cells expressing class MHC-II antigens, such as inflammatory cells, fibroblasts and vascular endothelial cells, and then transforms those various cells into antigen presenting cells (APC). So that denatured protein produces new antigenicity, which starts the humoral immunity and cell immunity resulting in aggravating corneal injury and corneal graft melting [41].

\section{Systemic diseases}

The incidence of corneal graft melting is higher in patients with Stevens - Johnson syndrome (SJS), mucous membrane pemphigoid or other rheumatic related diseases. Half of patients occurred sterile corneal stromal necrosis secondary to corneal graft melting [42,43].

Zou [44] believed that rheumatic diseases can damage the ocular surface and the reason for corneoscleral melting might be related to corneal marginal microvasculitis. However, the relevant specific mechanism for the cause not yet clearly described.

Manchanda et al. [45] showed that inhibitors of IL-1 $\beta$ and IL-1 receptors play an important role in the survival time of corneal graft. There was an endocrine cycle between TGF- $\beta 1$ and IL- $\beta$, and TGF- $\beta 1$ was used to reduce IL- $\beta$ in corneal graft, and the survival time of graft was prolonged [46,47]. Abnormal expression of TNF- $\alpha$ will suppress angiogenesis, dose-dependently activate MMP-9, and lead to corneal epithelial defect. MMP-9 can degrade gelatin, IV and V type collagen and elastin. The expression of MMP-9 significantly increases in corneal graft, while MMP-9 does not exist in the normal cornea. MMP-9 is positively associated with inflammation, infiltration of immune components and the intensity of graft rejection. MMP-9 can make corneal epithelial basement membrane dissolve [48-52]. MMP-2, which has a similar effect with MMP-9, will further dissolve the corneal stroma. A large number of MMP-2 that are released into stroma reduce a series of side reactions. Firstly, MMP-2 can activate protein cleavage reaction through the reconstruction of the ECM and basement membrane, MMP-2 can destroy the junctions between keratocytes and switch the function of extracellular matrix (ECM) adhesion to promote the differentiation of epithelial cells or the migration of endothelial cells. All those reactions result in delayed corneal epithelial healing, stromal edema, the infiltration of inflammatory cells, keratocytes reduction, massive cellulose deposition, angiogenesis, and ultimately corneal thinning and dissolution $[53,54]$. The inhibitory effect of the proteolytic system in the 
corneal stroma is weakened and collagenase is released by inflammatory cells located on the limbus, which dissolves the cornea $[44,55]$. The risk of grafts melting is great among some patients with spontaneous corneal dissolution is very great. In severe cases, planting bed even melts. Though MMP inhibition down-regulates the expression of MMP-2 and MMP-9, the survival time of graft was significantly prolonged [56].

\section{Surgical factors}

Retransplantation: Patients with repeat surgery are prone to immune rejection. Especially retransplantation leads to a diversity of graft antigens or an increase in the number, which aggravates neovascularization and immune reaction.

Some patients suffered from graft versus host disease after receiving other organ transplantation, which was often accompanying with eye injury. Corneal graft melting was likely to occur when such patients underwent keratoplasy [34].

Li et al. [20] analyzed 97 patients who had underwent keratoplasy and found 8 cases of corneal graft melting and aqueous leakage. Most of the patients had failed surgery for times and attempted keratoprosthesis. Because the patients had poor corneal condition and their corneas became thinner after surgery, it was prone to occurs corneal graft melting and perforation.

Combined surgery: Reports have confirmed that surgery can cause apoptosis or necrosis of normal corneal cell, followed by severe complications such as corneal graft melting and perforation [57]. The graft rejection rate is high after keratoplasy combined with other surgery. The severe patients undergo corneal graft melting, perforation or donor disk dislocation.

\section{Drug factors}

Non-steroidal anti-inflammatory eye drops (NSAIDs): Many reports have showed that NSAIDs can cause corneal graft melting. The mechanisms of NSAIDs-induced corneal graft melting as following: (1) NSAIDs inhibit cyclooxygenase enzymes (COX), thereby prevent peanut four acid from converting into prostaglandins. Thus, Peanut four acid converts to leukotriene. Leukotrienes can involve leukocyte to damage cornea and stimulate the degranulation of leukocyte to cause corneal graft melting. (2) NSAIDs disrupt the expression balance of MMP involved in extracellular matrix deposition and degeneration, while NSAIDs also increase the expression of MMPs [58]. Then MMPs degrade corneal stroma and prevent corneal epithelial wound healing, and then lead to corneal graft melting. Reviglio et al. [59] found that the expression of MMP-1 and MMP-8 was higher in the cornea in the diclofenac sodium group than in the ketorolac and artificial tears groups. A patient underwent corneal perforation because of diclofenac treatment about two months after PRK. It is observed that type III and IV collagen, MMP-3, and MMP-9 were accumulated in the wounded anterior stroma [60]. (3) NSAIDs affect corneal sensitivity by inhibiting the neuronal ion pathway, resulting in the loss of neurotrophic support to cornea. The use of NSAIDs after eye surgery resulted in destruction of corneal sensitivity [61]. (4) NSAIDs have a toxic effect on the cornea [62-64]. NSAIDS (especially diclofenac sodium) can cause apoptosis, which have been proved in animal experiments [58]. In vitro cellular researches showed that diclofenac sodium could reduce cell viability. Cellular toxicity after contacting to drugs increased, relating to drug concentration and contact time. Abnormal electrolyte composition, $\mathrm{pH}$ and osmolarity that were involved by NSAIDs, and the preservatives used in ophthalmic solutions can damage ocular surface, which will change cell condition [65].

Corticosteroid eye drops: Corticosteroid is commonly used after keratoplasy to prevent immune rejection. However, it has been reported that corticosteroid contributes to corneal graft melting. The mechanism may be as follows: (1) Corticosteroid is toxic to cells in a time- and dose-dependent manner. It can decrease cell viability, which inhibits fibroblasts proliferationfollowed by inhibiting corneal reepithelialization and the reconstruction of epithelial cells and matrix collagenous fiber [66]. (2) Corticosteroid can activate collagenase and suppresses the synthesis of collagen and proteoglycan $[67,68]$. Those methods delay corneal wound healing, make persistent corneal epithelial defects, might even speed up corneal graft melting. (3) The above abnormal changes of the ocular surface increase the risk of corneal infection and some patients develop corneal ulcer, corneal graft melting and perforation $[69,70]$.

Other uncommon drug: $\mathrm{Wu}$ et al. [71] reported a rare case about drug-induced corneal graft melting as Mooren-like ulcer, which frequently used topical anesthetics (lidocaine) and glucocorticoid eye drops, finally whose cornea was almost dissolved. On one hand, topical anesthetics decrease corneal sensitivity and prolong the exposure time of cornea and air, leading to secondary corneal necrosis, melting and perforation. On the other hand, Topical anesthetics may change corneal antigens that cause immune reactions to attack the cornea $[71,72]$.

\section{Materials}

After the implantation of biomaterials, the interaction between the host and biomaterials firstly occurs on the surface of the materials, and the surface property of the materials directly affects the protein adsorption, cell adhesion, proliferation and differentiation.

The solid back plate of keratoprosthesis made from early biological materials without permeability such as polymethylmethacrylate (PMMA), is unfavorable for the extracellular matrix to enter keratoprosthesis. It has been reported that delayed hypersensitivity is associated with KPro materials with high expression of inflammatory cytokines [73]. A research showed that the corneal graft melting happened in $51 \%$ of eyes equipped with conventional keratoprosthesis, comparing with $10 \%$ of eyes equipped with new-type keratoprosthesis. Epithelial cells are unable to grow and cover keratoprosthesis. As a result, the necrosis of the periphery of keratoprosthesis is prone to occur owing to the absence of innervation. Once it occurs, keratoprosthesis cannot be 
combined with the planting bed, resulting in tissue necrosis or graft melting [74]. Some researchers think that collagenase secreted by corneal epithelium plays an important role in corneal graft melting and collagenase inhibitors can inhibit this process. The incompatibility between biomaterials and planting bed can cause aseptic necrosis and matrix lysis around the graft. Since the improvement of biocompatibility, the materials can better adhere to the planting bed, and the corneal graft melting rate has reduced [75].

PHEMA has good flexibility and high-water content, and it is wildly used. The central optic and a biointegrating anchoring skirt of AlphaCor keratoprosthesis are composed of PHEMA. Postoperative corneal stromal melting of AlphaCor Keratoprothesis occurs between the peripheral skirt and planting bed. Vijayasekaran et al. [76] showed that polymorphonuclear neutrophils (PMNs) firstly appeared in the graft and tissue within $24 \mathrm{~h}$ postoperatively. Macrophages appeared in the tissue, most of which was in the graft/tissue interface within two weeks. There were large numbers of macrophages and neutrophils in the graft within three months [77].

The histological experiments showed that PHEMA released tissue factor in response to postoperative reaction. Host tissue products, enzymes and many inflammatory cytokines gather in the device that damages the cornea during the healing of surgical incision. If it overreacts, it may cause instability of biomaterials and then cause corneal graft melting [78-80].

Nonwoven polypropylene and polyurethan as scaffold have poor healing effects to the cornea. Even though corneal stroma and keratocytes can grow into the scaffold, it still cannot avoid tissue melting. Finally, the scaffold will be exposed, and it needs to replace Keratoprothesis.

The main materials used for tissue-engineered corneal scaffolds are acellular porcine corneal stroma, collagen, amniotic membrane, chitosan and so on. The Unsmooth surface of corneal planting bed, hematocele and suture loosening of graft are main reasons of dissolution of the amnion membrane, which mechanism has not been analyzed in detail [81]. Collagen is degraded rapidly and can be digested by collagenase [82]. Other biological materials have not been specifically reported about corneal graft melting.

\section{Prognosis}

More than half of patients who have undergone corneal graft melting after keratoplasy can achieve corneal epithelialization in the end. Most of the patients with corneal graft melting become steady after treatment of observation, amniotic membrane covering or transplantation, autogenous conjunctival flap covering, and medical treatment. Some patients need repeat keratoplasy or corneal repair. In addition, almost half KPro-related melt also can be resolved by artificial corneal exchange [83-85]. A few severe patients who continues to deteriorate with or without management develop endophthalmitis and enucleation of eyeballs [2,27].
In research of $\mathrm{Li}$, all patients with sterile stromal melting after DALK could eventually turn to corneal epithelialization or neovascularization after timely treatment [27].

Cases of severe fungal keratitis undertook penetrating keratoplasty by Wang et al., and 8 cases (11\%) recurred [86]. Two of fifty patients had undergone MICOF keratoprosthesis happened superficial corneal graft melting around column mirror. One eye healed spontaneously, while other one healed by autogenous periosteum repairing and conjunctival covering.

The incidence of Melt-related NLP acuity is $42.1 \%$ after keratoplasy, $26.3 \%$ of which is main reason. Also, corneal graft melting can lead to the device extrusions (KPro-related melting), endophthalmitis, and choroidal detachments.

\section{Prevention}

There are many reasons for postoperative corneal graft melting after keratoplasy. It is also possible that multiple causes lead to corneal graft melting. Therefore, comprehensive analysis and combination therapy are often required.

\section{Remove the risk factors}

For the patients with vascularization of corneal planting bed, glucocorticoid eye drops, NSAIDs and vitamin $\mathrm{C}$ are commonly used drugs for the corneal neovascularization. But glucocorticoid eye drops and NSAIDs should be used appropriately. VEGF inhibitors, apoptosis inducing factors, plasminogens, peroxidase 6 are being studied, and gene therapy is the direction of future research [36]. Some studies have shown that using bevacizumab locally or under conjunctiva can inhibit rejection and cause partial neovascularization to degenerate [87]. Gene therapy is to integrate DNA into the target cells and ensure the accurate localization of DNA, sustained expression of gene, which will avoid immune response or induced-pathogenicity. The carrier plays an important role in keratoplasty and it can be either transported to the donor grafts in vitro or to the recipient in vivo [88].

For the patients with poor postoperative corneal healing, treatments include re-stitching or covering amniotic membrane. Mild melting can recover by treatment of adequate lubrication, such as artificial tears. Punctal occulusion and bandage contact lens are applicable for corneal graft melting by various factors. It can increase the moisture of the corneal surface, thereby reduce the loss of corneal epithelium and the incidence of corneal graft melting, which can prolong the retention time of implants [89]. Most patients with chemical injury who are likely to have limbal stem cell deficiency can achieve satisfactory results after managements of tarsorrhaphy, amniotic membrane graft, conjunctival flap graft or corneal limbal stem cell autograft transplantation [27].

\section{Treat the primary diseases}

Due to the characteristics of herpes simplex virus and fungi, the lesion has to be completely removed away to protect from recurrence in the process of keratoplasty. The hypopyon and 
fibrous exudative membrane should be completely removed [2]. Antiviral or antifungal agents are respectively continued to administer after the operation. For the patients with fungal infection recurrence, the glucocorticoid eye drops should be forbidden within 2 weeks after operation. If there are no signs of primary recurrence after 2 weeks, glucocorticoid eye drops can be experimentally used [90].

\section{Use Immunosuppressants and glucocorticoids}

Cyclosporine A and FK506 are common Immunosuppressive ophthalmic preparations that can effectively prevent neovascularization and release of inflammatory factors, and induce the tolerance of the body to the foreign antigen; therefore, inhibit the immune response [91,92]. Corneal graft melting that occurs in the patients with autoimmune diseases after keratoplasy is difficult to control by general treatments. Therefore, glucocorticoid and immunosuppressants should be administrated before keratoplasy, and also a systemic treatment of the primary diseases and further melting [93]. Shan et al. [93] used oral methotrexate and prednisone topical cyclosporine, and medroxyprogesterone (MPG) to treat a patient who still worse after general treatment, and finally ulcer healed with poor vision.

\section{Symptomatic treatment}

Matrix metalloproteinases are associated with corneal graft melting and anti-collagenase drug may protect cornea from further melting. If the cornea melts further or other treatments are ineffective, repeated keratoplasty is required and artificial cornea exchange or replacement with a donor cornea [18].

It should actively prevent from postoperative corneal graft melting. Primary disease and ocular inflammation should be controlled prior to surgery. At the same time, it is feasible to reinforce ocular surface with autogenous periosteum, autogenous bulbar fascia, the conjunctival flap and amniotic membrane graft for those patients with corneal weakness, neovascularization or blepharodiastasis. Permanently suturing lateral one third of lid fissure can reduce corneal exposure area, reduce tear evaporation, and therefore prevent corneal graft melting [94].

\section{Summary}

However, in keratoplasty, the incidence of corneal graft melting is lesser than the other complication, higher attention should be needed to avoid consequences. Preoperative detailed ocular and systemic examinations are important, and also effective time management in treatment administration is required after surgery. In conclusion, analyses of the risk factors of corneal graft melting may provide targeted therapies to improve the outcomes of keratoplasy.

\section{Conflict of Interest}

No conflicting relationship exists for any author.

\section{References}

1. Huber KK, Maier AK, Klamann MK, et al. Glaucoma in penetrating keratoplasty: risk factors, management and outcome. Graefes Arch Clin Exp Ophthalmol. 2013;251:105-16.

2. Zhao G, Jiang $\mathrm{N}, \mathrm{Hu}$ L. Penetrating keratoplasty for treatment of high-risk fungal keratitis. Journal of Clinical Rehabilitative Tissue Engineering Research. 2009; 13:3597-600.

3. Krysik K, Dobrowolski D, Lyssek-Boron A, et al. Differences in Surgical Management of Corneal Perforations, Measured over Six Years. J Ophthalmol. 2017;2017:1582532.

4. Spadea L, Abbouda A, Abicca I, et al. Aspergillus flavus endophthalmitis after penetrating keratoplasty combined with cataract phacoemulsification and IOL implantation. Int Ophthalmol. 2015;35:145-8.

5. Jastaneiah SS, Al-Rajhi AA, Abbott D. Ocular mycosis at a referral center in Saudi Arabia: A 20-year study. Saudi J Ophthalmol. 2011;25:231-8.

6. Schaub F, Bachmann BO, Seyeddain O, et al. Mid- and Longterm Experiences with the Boston-Keratoprosthesis. The Cologne and Salzburg Perspective. Klin Monbl Augenheilkd. 2017;234:770-5.

7. Nau AC, Drexler S, Dhaliwal DK, et al. Contact lens fitting and long-term management for the Boston keratoprosthesis. Eye Contact Lens. 2014;40:185-9.

8. Chan CC, LoVerde L, Qiang J, et al. Incidence, Risk Factors, and Surgical Management of Boston Type 1 Keratoprothesis Corneal Melts, Leaks, and Extrusions. Cornea. 2016;35: 1049-56.

9. Chan CC, Holland EJ. Infectious keratitis after Boston type 1 keratoprosthesis implantation. Cornea. 2012;31:1128-34.

10. Robert MC, Dohlman CH. A review of corneal melting after Boston Keratoprosthesis. Semin Ophthalmol. 2014;29:349-57.

11. Kim MJ, Yu F, Aldave AJ. Microbial keratitis after Boston type I keratoprosthesis implantation: incidence, organisms, risk factors, and outcomes. Ophthalmology. 2013;120:2209-16.

12. Duan D, Klenkler BJ, Sheardown H. Progress in the development of a corneal replacement: keratoprostheses and tissue-engineered corneas. Expert review of medical devices. 2006;3:59-72.

13. Canwei Z. Advances in the construction of tissueengineered cornea in vitro. Chinese Journal of Experimental Ophthalmology. 2017;35:170-4.

14. Zhang MC, Liu X, Jin Y, et al. Lamellar keratoplasty treatment of fungal corneal ulcers with acellular porcine corneal stroma. Am J Transplant. 2015;15:1068-75.

15. Ma XY, Zhang Y, Zhu D, et al. Corneal Stroma Regeneration with Acellular Corneal Stroma Sheets and Keratocytes in a Rabbit Model. PloS one. 2015; 10:e0132705. 
16. Liu X-N, Zhu X-P, Wu J, et al. Acellular ostrich corneal stroma used as scaffold for construction of tissueengineered cornea. Int J Ophthalmol. 2016;9:325-31.

17. Aldave AJ, Sangwan VS, Basu S, et al. International results with the Boston type I keratoprosthesis. Ophthalmology. 2012;119:1530-8.

18. Utine CA., Tzu JH, Akpek EK. Clinical features and prognosis of Boston type I keratoprosthesis-associated corneal melt. Ocul Immunol Inflamm. 2011;19:413-8.

19. Greiner MA, Li JY, Mannis MJ. Longer-term vision outcomes and complications with the Boston type 1 keratoprosthesis at the University of California, Davis. Ophthalmology. 2011;118:1543-50.

20. Li L, Yifei H, Jifeng Y. Causes and nursing care of postoperative complications in patients receiving keratoprosthesis. Chinese Journal of Nursing. 2014;47:225-6.

21. Yan L, Zouhe L, Lan L. Experience in Constructin Rat Model of Penetrating Keratoplasty. Laboratory Animal Science and Administration. 2002;19:8-11.

22. Zerbe BL, Belin MW, Ciolino JB. Results from the multicenter Boston Type 1 Keratoprosthesis Study. Ophthalmology. 2006;113:1779 e1771-7.

23. Chew HF, Ayres BD, Hammersmith KM, et al. Boston keratoprosthesis outcomes and complications. Cornea. 2009;28:989-96.

24. Yaghouti F, Nouri M, Abad JC, et al. Keratoprosthesis: preoperative prognostic categories. Cornea. 2001;20:19-23. 27.

25. Kaiwen H, Yan H, Tingting W. Preliminary study of dexamethasone's curative effect of acid burns on rat cornea. International Eye Science. 2014;3:416-8.

26. Chengbo F, Ti C, Yongjian Y. The changes of SOD and MDA in cornea and aqueous humour after photorefractive keratectomy. Chinese Ophthalmic Research. 2003;21:341-3.

27. Li S, Wei C, Huixiang M. Complications and treatment for deep anterior lamellar keratoplasty. Chinese Journal of Optometry Ophthalmology and Visual Science. 2012;14:684-7.

28. Hong J, Peng R, Hao Y. [Post operation complications analysis and their treatment of Descemet's stripping automated endothelium keratoplasty]. Chinese Journal of Ophthalmology. 2014;50:254-60.

29. Liansheng W, Jiansu C, Jintang X. Cat xenogeneic lamellar keratoplasty for re-construction of corneal surface after severe corneal alkali burn in rabbits. Recent Advances In Ophthalmology. 2010;30:105-8.

30. Niederer RL, Perumal D, Sherwin T. Corneal innervation and cellular changes after corneal transplantation: an in vivo confocal microscopy study. Invest Ophthalmol Vis Sci. 2007;8:621-6.

31. Na W, Rui Z, Fengyuan S. Dry eye syndrome after penetrating keratoplasty. Chinese Journal of Ocular Trauma and Occupational Eye Disease. 2013;35:215-7.
32. Deqi Y, Jing H. Research on the tear film function after keratoplasty. International journal of ophthalmology. 2008;8:1200-2.

33. Xiuli S, Weiyun S, Ting W. Factors associated with delayed epithelial healing in early stage after lamellar keratoplasty. Journal of Clinical Ophthalmology. 2013;21:97-100.

34. Liuhe Z. Treatment and prevention of corneal graft melting after keratoplasty. Ophthalmology In China. 2009;8:148-9.

35. Lyall DA, Tarafdar S, Gilhooly MJ, et al. Long term visual outcomes, graft survival and complications of deep anterior lamellar keratoplasty in patients with herpes simplex related corneal scarring. $\mathrm{Br} \mathrm{J}$ Ophthalmol. 2012;96:1200-3.

36. Huan L, Jinling L, Xiaorong Z. Investigation about the use of drug after keratoplasty complications. Technology Wind. 2016;2016:55-9.

37. Yingce F. An Analysis of Corneal Ulcer Debridement Combined with Conjunctiva Flap Covering in Treatment of Fungal Corneal Ulcer. Guide of China Medicine. 2015;2015:13-4.

38. Meixia R. Matrix metalloproteinases/tissue inhibitors of metalloproteinase system and diabetic ocular complications. Chinese Journal of Experimental Ophthalmology. 2014;32:860-4.

39. Xiaoyan X, Shanbi Z, Qi Z. Clinical research of repeat keratoplasty. Chinese Journal of Practical Ophthalmology. 2013;31:331-6.

40. Hanping X, Jiaqi C, Xiongfei G. A primary study on soluble protein and soluble antigen of corneal stroma in Mooren's ulcer patients. Chinese Journal Of Ophthalmology. 2002;38:360-2.

41. Zhengmao $X$. Research advance in immunological mechanism after corneal alkali burns. Chinese Ophthalmic Research. 2010;28:796-9.

42. Alexander JK, Basak SK, Padilla MD, et al. International Outcomes of the Boston Type I Keratoprosthesis in Stevens-Johnson Syndrome. Cornea. 2015;34:1387-94.

43. Robert MC, Arafat SN, Ciolino JB. Collagen cross-linking of the Boston keratoprosthesis donor carrier to prevent corneal melting in high-risk patients. Eye Contact Lens. 2014;40: 376-81.

44. Liuhe Z. Importance of recognition of ocular surface manifestation of rheumatic diseases. Ophthalmology In China. 2006;15:159-60.

45. Manchanda PK, Bid HK, Kumar A, et al. Genetic association of interleukin-1beta and receptor antagonist (IL-1Ra) gene polymorphism with allograft function in renal transplant patients. Transpl Immunol. 2006;15:289-96.

46. Aoki H, Ohnishi H, Hama K, et al. Autocrine loop between TGF-betal and IL-1beta through Smad3- and ERK-dependent pathways in rat pancreatic stellate cells. Am J Physiol Cell Physiol. 2006;290:C1100-8. 
47. Puning Z, Jing L, Liehong Z. Analysis of high risk factors associated with graft rejection after penetrating keratoplasty. China Tropical Medicine. 2007;7:74-5.

48. Rigal-Sastourne JC. Corneal burns and matrix metalloproteinases (MMP-2 and -9): the effects of human amniotic membrane transplantation. J Fr Ophtalmol. 2002 25, 685-693 (2002).

49. C.J, Y., M, D. \& J, H. Expression of MMP-2 and MMP-9 in corneal thermal burn. International journal of ophthalmology 8, 1773-1776 (2008).

50. Isnard N, Robert L, Renard G. Effect of sulfated GAGs on the expression and activation of MMP-2 and MMP-9 in corneal and dermal explant cultures. Cell Biol Int. 2003;27:779-84.

51. Shi QC, Ning JH. Restrained effect of GM-6001 the tissue inhibitor of matrix metalloproteinases on the growth of rat's corneal neovascularization after alkaline burn. International journal of ophthalmology. 2008;8:496-8.

52. Fang L, Yiqiao X, Yanning Y. Expression of matrix metalloproteinase 9 and tissue inhibitor of metalloproteinase 1 in mouse cornea with alkali burn. Recent Advances In Ophthalmology. 2005;25: 309-311.

53. Lafleur MA, Forsyth PA, Atkinson SJ, et al. Perivascular cells regulate endothelial membrane type-1 matrix metalloproteinase activity. Biochem Biophys Res Commun. 2001;282:463-73.

54. Li L, Zhou D, Xiumei W. Expression of matrix metalloproteinases and inhibitor on the cornea tissue in rabbit after implantation of modified titanium skirt for keratoprosthesis. Chinese Journal of Ophthalmology. 2012;48:20-6.

55. Antao SF, Ayoub T, Tahir H, et al. Stabilization of bilateral progressive rheumatoid corneal melt with infliximab. Case Rep Ophthalmol Med. 2012;2012:173793.

56. Eaton VL, Lerret NM, Velásquez-Lopera MM, et al. Enhanced allograft survival and modulation of T-cell alloreactivity induced by inhibition of MMP/ADAM enzymatic activity. Am J Transplant. 2008;8:507-16.

57. Engelsvold DH, Utheim TP, Olstad OK, et al. miRNA and mRNA expression profiling identifies members of the miR-200 family as potential regulators of epithelialmesenchymal transition in pterygium. Exp Eye Res. 2013;115:189-98.

58. Juan L, Haodong Z, Jiwei T. The Relationship between the Corneal Melting and Nonsteroidal Anti-inflammatory Drug after Excision of Pterygium. Medical Information. 2014;2014:72.

59. Reviglio VE, Rana TS, Li QJ, et al. Effects of topical nonsteroidal antiinflammatory drugs on the expression of matrix metalloproteinases in the cornea. J Cataract Refract Surg. 2003;29:989-97.

60. Gabison EE, Chastang P, Menashi S, et al. Late corneal perforation after photorefractive keratectomy associated with topical diclofenac: involvement of matrix metalloproteinases. Ophthalmology. 2003;10:1626-31.

61. Lin JC, Rapuano CJ, Laibson PR, et al. Corneal melting associated with use of topical nonsteroidal anti- inflammatory drugs after ocular surgery. Arch Ophthalmol. 2000;118:1129-32.

62. Xiaobin Z, Xiaoming Y. Nonsteroidal anti-inflammatory eyedrops and corneal melting. International Review Of Ophthalmology. 2006;30:394-7.

63. Yue Z, Shi Z, Jinhong C. Ultrastructural pathological alterations of corneal organization by local application of corticosteroid and NSAIDs in rabbits. Chinese Journal Of Practical Ophthalmology. 2011;29:281-4.

64. Mingli Q, Haoyun D, Yao W. Cytotoxicity research of three non-steroidal anti-inflammatory eye drops in human corneal epithelial cells. Chinese Journal of Experimental Ophthalmology. 2015;33:627-32.

65. Lee JS, Kim YH, Park YM. The Toxicity of Nonsteroidal Anti-inflammatory Eye Drops against Human Corneal Epithelial Cells in Vitro. J Korean Med Sci. 2015;30:1856-64.

66. Xiaowen S, Xiuli B, Danfu C. Clinical analysis of six cases of scleral melting after pterygium surgery. Chinese Journal of ocular trauma and occupational eye disease; 2015;37:711-3.

67. Piper SL, Laron D, Manzano G, et al. A comparison of lidocaine, ropivacaine and dexamethasone toxicity on bovine tenocytes in culture. J Bone Joint Surg Br. 2013;94:856-62.

68. Zhang JZ, Krenzer KL, Lopez FJ, et al. Comparative effects of besifloxacin and other fluoroquinolones on corneal reepithelialization in the rabbit. J Cataract Refract Surg. 2010;36:1049-50.

69. Lichao W, Wei Z. Prevention and therapy progress in allograft rejection of high - risk corneal transplantation. International Eye Science. 2014;14:1413-6.

70. Fasolo A, Pedrotti E, Passilongo M, et al. Safety outcomes and long-term effectiveness of ex vivo autologous cultured limbal epithelial transplantation for limbal stem cell deficiency. Br J Ophthalmol. 2017;101:640-9.

71. Hui W, Yuan H, Xiaoru S. Keratopathy due to ophthalmic drug abuse with corneal melting and perforation presenting as Mooren-like ulcer: A case report. Experimental And Therapeutic Medicine. 2016;12:343-6.

72. Khakshoor H, Moshirfar M, Simpson RG, et al. Anesthetic keratopathy presenting as bilateral Mooren-like ulcers. Clinical Ophthalmology. 2012;6:1719-22.

73. Coassin M, Zhang C, Green WR, et al. Histopathologic and immunologic aspects of alphacor artificial corneal failure. Am J Ophthalmol. 2007;144:699-704.

74. Jia C. Advancement of keratoprosthesis research. International Journal of Ophthalmology. 2008;28:1279-84.

75. Kirkham SM, Dangel ME. The keratoprosthesis: improved biocompatibility through design and surface modification. Ophthalmic Surg. 1991;22:455-61.

76. Vijayasekaran S, Fitton JH, Hicks CR, et al. Cell viability and inflammatory response in hydrogel sponges implanted in the rabbit cornea. Biomaterials. 1998;19:2255-67.

77. Vince DG, Hunt JA, Williams DF. Quantitative assessment of the tissue response to implanted biomaterials. Biomaterials. 1991;12:731-6. 
78. Kanayama S, Garty S, Kim B, et al. Histological study of graft failure in AlphaCor transplantation. International ophthalmology. 2011;31:501-4.

79. Jiraskova N, Werner L, Mamalis N, et al. Histologic evaluation of AlphaCor keratoprosthesis explanted following various complications. Biomedical papers of the Medical Faculty of the University Palacky, Olomouc, Czechoslovakia. 2014;158:50-5.

80. Ruijie M, Yan L, Zhulin H. Research progress on scaffold materials and biocompatibility of keratoprosthesis. International Review of Ophthalmology. 2016;40:217-21.

81. Jiao L, Linbin W, Daili X. Analysis of the failure reason of amniotic membrane transplantation in treatment of pterygium. International journal of ophthalmology. 2011;11:1282-3.

82. Qian D. Preparation and biocompatibility of chitosancollagen corneal repair materials. Chinese Journal of Tissue Engineering Research. 2015;2015:8433-7.

83. Liqiang W, Hua B, Yifei, H. Analysis of clinic results and complication associated with Boston keratoprosthesis. Chinese Journal of Ophthalmology. 2015;51:673-6.

84. Lei Z, Liya W, Yueqin Z. Long-term outcomes of Boston type keratoprosthesis for Chinese severe corneal blindness. Chinese Journal of Experimental Ophthalmology. 2015;33:930-4.

85. Bouhout S, Robert MC, Deli S, et al. Corneal Melt after Boston Keratoprosthesis: Clinical Presentation, Management, Outcomes and Risk Factor Analysis. Ocul Immunol Inflamm. 2017;2017:1-7.

86. Yuexin W, Dai W, Yangyang Z. The regularity of recurrent and immune rejection after large-diameter penetrating keratoplasty for the treatment of fungal keratitis. Chinese Journal of Optometry Ophthalmology and Visual Science. 2015;17:685-9.

87. Patsoukis N, Sari D, Boussiotis VA. PD-1 inhibits T cell proliferation by upregulating $\mathrm{p} 27$ and $\mathrm{p} 15$ and suppressing Cdc25A. Cell cycle. 2012;11:4305-9.
88. Di Z. Gene Therapy of Corneal Graft Rejection. Medical Recapitulate. 2016;22:216-20.

89. Zarei-Ghanavati M, Avadhanam V, Vasquez Perez A, et al. The osteo-odonto-keratoprosthesis. Current opinion in ophthalmology. 2017;28:397-402.

90. Qian Z, Ting W, Weiyun S. Treatment and prognosis of recurrent fungal keratitis after corneal transplantation. Chinese Journal of Experimental Ophthalmology. 2015;33:338-41.

91. Kim DW, Lee SH, Shin MJ, et al. PEP-1-FK506BP inhibits alkali burn-induced corneal inflammation on the rat model of corneal alkali injury. BMB Rep. 2015;48:618-23.

92. Fuqiang L, Yu L, Yahong X. Effects of cyclosporin A on aqueous fluid inflammatory cytokines in high-risk corneal transplantation patients. Journal of Clinical Ophthalmology. 2016;24:203-6.

93. Shan SJ, Wu EI, Akpek EK. Sterile corneal melt after descemet stripping endothelial keratoplasty in patients with previously undiagnosed Sjogren syndrome. Arch Ophthalmol. 2009;127:219-20.

94. Yifei H, Liqiang W, Fengxiang W. Clinical application of keratoprosthesis for corneal opacity unsuitable for keratoplasty. Chinese Journal Of Ophthalmology. 2003;39:578-81.

\section{*Correspondence to}

Xiuming Bin

Eye Center, Affiliated Second Hospital

Zhejiang UniversityHangzhou 310009, China.

E-mail: lzyjxm@zju.edu.cn 